\title{
Updated analysis of studies on the cytotoxic T-lymphocyte-associated antigen-4 gene A49G polymorphism and Hashimoto's thyroiditis risk
}

\author{
R. Ji ${ }^{1}$, Y. Feng ${ }^{2}$ and W.W. Zhan ${ }^{1}$ \\ ${ }^{1}$ Department of Ultrasonography, School of Medicine, Ruijin Hospital, \\ Shanghai Jiaotong University, Shanghai, China \\ ${ }^{2}$ Department of Respiration, School of Medicine, Ruijin Hospital, \\ Shanghai Jiaotong University, Shanghai, China \\ Corresponding authors: Y. Feng / W.W. Zhan \\ E-mail: hywan_fy@yahoo.com.cn
}

Genet. Mol. Res. 12 (2): 1421-1430 (2013)

Received July 23, 2012

Accepted December 17, 2012

Published April 26, 2013

DOI http://dx.doi.org/10.4238/2013.April.26.4

\begin{abstract}
Published data on the association between the cytotoxic T-lymphocyte-associated antigen-4 gene A49G polymorphism and the risk for Hashimoto's thyroiditis (HT) are inconclusive. A meta-analysis was performed to derive a more precise estimation. Published casecontrol studies in English or Chinese were identified. In total, 24 studies with 2295 cases and 4521 controls were investigated. A random-effect model was performed irrespective of between-study heterogeneity. Study quality was assessed in duplicate, and subgroup analyses were conducted by ethnicity or age. Overall, the $49 \mathrm{G}$ allele was associated with an increase in HT risk [odds ratio $(\mathrm{OR})=1.31 ; 95 \%$ confidence interval $(95 \% \mathrm{CI})=1.17-1.47 ; \mathrm{P}<0.00001]$. In a subgroup analysis by ethnicity, comparison of allele $49 \mathrm{G}$ with $49 \mathrm{~A}$ generated a $27 \%$ increased risk among East Asians $(\mathrm{OR}=1.48 ; 95 \% \mathrm{CI}=1.24-1.76 ; \mathrm{P}<0.00001)$ and whites $(\mathrm{OR}=1.27 ; 95 \% \mathrm{CI}=1.12-1.44 ; \mathrm{P}=0.0002)$. We also found an increased risk among adults $(\mathrm{OR}=1.31 ; 95 \% \mathrm{CI}=1.17-1.47 ; \mathrm{P}<$ $0.00001)$ but not among children $(\mathrm{OR}=1.44 ; 95 \% \mathrm{CI}=0.75-2.79 ; \mathrm{P}=$ $0.27)$, possibly owing to the small sample sizes in children. No publication
\end{abstract}


biases were observed. This meta-analysis suggested that the cytotoxic T-lymphocyte-associated antigen-4 gene $49 \mathrm{G}$ allele was associated with an increased HT risk, especially in adults.

Key words: Meta-analysis; Hashimoto's thyroiditis; $C T L A-4$; A49G polymorphism

\section{INTRODUCTION}

Hashimoto's thyroiditis (HT) is one of the most common human autoimmune thyroid diseases. In HT, the antibodies against thyroid peroxidase or thyroglobulin appear characteristically in the sera of patients, and tissue damage owing to T-cell-mediated cytotoxicity usually contributes to the gradual development of hypothyroidism (Weetman, 2003). Several reports have demonstrated that multiple genetic factors are responsible for the development of HT, and the cytotoxic T-lymphocyte-associated antigen-4 (CTLA-4) gene has been extensively studied.

CTLA-4 (CD152), which is expressed on the surface of activated T lymphocytes, is a negative regulator of T-cell activation (Scheipers and Reiser, 1998). Evidence is accumulating that the CTLA-4 gene is associated with a variety of autoimmune conditions (Tomer, 2001), such as autoimmune thyroid diseases (Graves' disease, HT, and thyroid antibodies) (Ban et al., 2003), Addison's disease (Donner et al., 1997), insulin-dependent diabetes mellitus (Kavvoura and Ioannidis, 2005; Baniasadi et al., 2006), systemic lupus erythematosus (Lee et al., 2005), and systemic sclerosis (Almasi et al., 2006). The CTLA-4 gene is located on 2q33, where an $\mathrm{A} / \mathrm{G}$ single nucleotide polymorphism at position 49 (exon 1, codon 17) has been identified.

Several case-control studies have investigated the association between the A49G polymorphism and HT, but they had low statistical power and their results were often not reproducible. To address this issue systematically, we performed a meta-analysis of all available case-control studies reported in English or Chinese to explore the association between the CTLA-4 gene A49G polymorphism and the risk for HT.

\section{MATERIAL AND METHODS}

\section{Publication search}

The electronic databases Medline (Ovid), PubMed, China National Knowledge Infrastructure, Wanfang, and Weipu were used to search for electronic publications that were published as of November 2011. The key words used for the search were "CTLA4 or CTLA-4 or cytotoxic T-cell lymphocyte associated antigen 4 or CD152" and "Hashimoto*" and "49 or A49G", combined with "gene or variant or polymorphism or allele". If multiple publications were available from the same study group, the most complete and recent results were abstracted. Search results were limited to articles published in English or Chinese and studies performed in human subjects.

\section{Inclusion/exclusion criteria}

Case-control studies were included in this meta-analysis, regardless of sample size, 
1) if they explored the association between the CTLA-4 gene A49G polymorphism and HT among unrelated subjects, 2) if genotyping was performed using validated methods, and 3) if they provided sufficient information on genotype or allele frequencies to allow an estimation of odds ratio (OR) and its corresponding 95\% confidence interval $(95 \% \mathrm{CI})$. HT was diagnosed by the presence of goiter, hypothyroidism, and elevated microsomal or thyroid peroxidase autoantibodies. Thyroid ultrasound showed reduced echogenicity.

\section{Data extraction}

Two reviewers (Y.F. and R.J.) independently checked all potentially relevant studies. The following data were collected from each study: first author, year of publication, ethnicity, study design, diagnostic criteria, baseline characteristics of the study population, total number of cases and controls, and genotype distributions in cases and controls. Information on the Hardy-Weinberg equilibrium (HWE) test was also tracked or calculated if unavailable. After data extraction, discrepancies were adjudicated by discussion and a consensus was reached.

\section{Statistical methods}

The meta-analysis was calculated using the Review Manager version 5.0.19 software [http://www.cc-ims.net/revman/download (accessed October 12, 2012)]. HWE was assessed with the Pearson chi-squared test or the Fisher exact test (SAS version 9.1.3, Institute Inc., Cary, NC, USA). No assumptions were required for genetic models of inheritance for the polymorphism under study.

Generally, the inconsistency index was used to examine the presence of betweenstudy heterogeneity with a statistical significance of 0.1 . In this study, we applied the randomeffect model for all comparisons because it accommodates the possibility that the underlying effect differs across studies. For practical use, the random-effect model is more conservative and has a wider $95 \% \mathrm{CI}$ than the fixed-effect model.

In addition, sensitivity analysis or subgroup analysis was performed to look at more narrowly drawn subsets of the studies by removing an individual study each time or studies with similar features such as deviation from HWE to assess their separate influence. Finally, we assessed publication bias using the fail-safe number $\left(N_{\mathrm{fs}}\right)$ with the significance set at 0.05 for each meta-comparison. Specifically, if the calculated $N_{\mathrm{fs}}$ value was smaller than the number of studies observed, the meta-analysis results might have publication bias. We calculated the $N_{\text {fs } 0.05}$ according to the formula $N_{\text {fs } 0.05}=\left(\sum Z / 1.64\right) 2-\mathrm{k}$, where $k$ is the number of articles included.

\section{RESULTS}

\section{Study inclusion and characteristics}

Based on our search strategy, the primary screening produced 33 potentially relevant articles, of which 24 studies met the inclusion criteria after evaluating the association of the A49G polymorphism with HT [Donner et al., 1997; Awata et al., 1998; Kouki et al., 2000; Park et al., 2000; Petrone et al., 2001; Wang et al., 2001; Tomoyose et al., 2002; Terauchi et al., 2003; Ueda et al., 2003; Zhou et al., 2003; Balbi et al., 2007; Kavvoura et 
al., 2007 (a meta-analysis, which included 3 unpublished studies: Ban et al.; Akazumi et al.; Ghaderi et al.); Dallos et al., 2008; Yesilkaya et al., 2008; Yu et al., 2008; Bicek et al., 2009; Kucharska et al., 2009; Sahin et al., 2009; Shi et al., 2010; Benhatchi et al., 2011; Yang et al., 2012; Farra et al., 2012]. In total, 2165 HT patients and 4284 controls were examined. Of these 24 articles, 17 were published in English (Donner et al., 1997; Awata et al., 1998; Kouki et al., 2000; Petrone et al., 2001; Tomoyose et al., 2002; Terauchi et al., 2003; Ueda et al., 2003; Balbi et al., 2007; Kavvoura et al., 2007; Dallos et al., 2008; Yesilkaya et al., 2008; Bicek et al., 2009; Kucharska et al., 2009; Sahin et al., 2009; Benhatchi et al., 2011; Yang et al., 2012; Farra et al., 2012), and 4 were in Chinese (Wang et al., 2001; Zhou et al., 2003; Yu et al., 2008; Shi et al., 2010). Three unpublished articles contained results of a study by Kavvoura et al. (2007). Additionally, the genotypes in controls for one case-control study were inconsistent with HWE (Shi et al., 2010). The detailed selection process is presented in Figure 1.

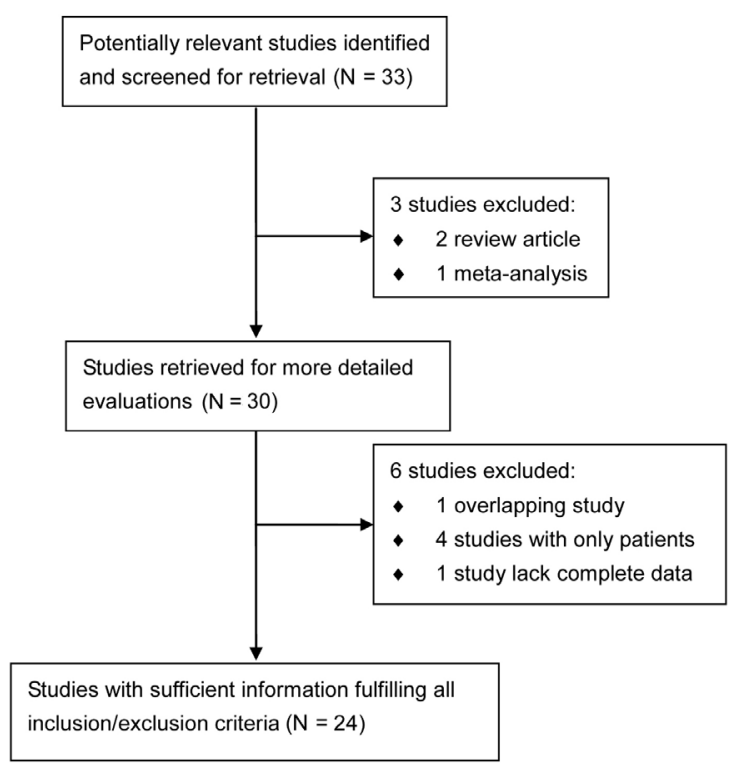

Figure 1. Flow diagram of search strategy and study selection.

The baseline characteristics of qualified studies are presented in Table 1. Of the 24 case-controls studies examining the association between the CTLA-4 A49G polymorphism and HT risk, 11 included East Asians [Awata et al., 1998; Park et al., 2000; Wang et al., 2001; Tomoyose et al., 2002; Terauchi et al., 2003; Zhou et al., 2003; Kavvoura et al., 2007 (a metaanalysis, which included 2 unpublished studies: Ban et al. and Ghaderi et al.); Yu et al., 2008; Shi et al., 2010; Yang et al., 2012]; 9 included whites (Donner et al., 1997; Kouki et al., 2000; Petrone et al., 2001; Ueda et al., 2003; Balbi et al., 2007; Dallos et al., 2008; Bicek et al., 2009; Kucharska et al., 2009; Benhatchi et al., 2011); 4 included the Middle Easterners: two studies that included Turks (Yesilkaya et al., 2008; Sahin et al., 2009), one that included Iranians (Kavvoura et al., 2007), and one that included Lebanese (Farra et al., 2012). Twenty-one studies were performed in adults, whereas 3 were in children (Dallos et al., 2008; Yesilkaya et al., 2008; Kucharska et al., 2009). 


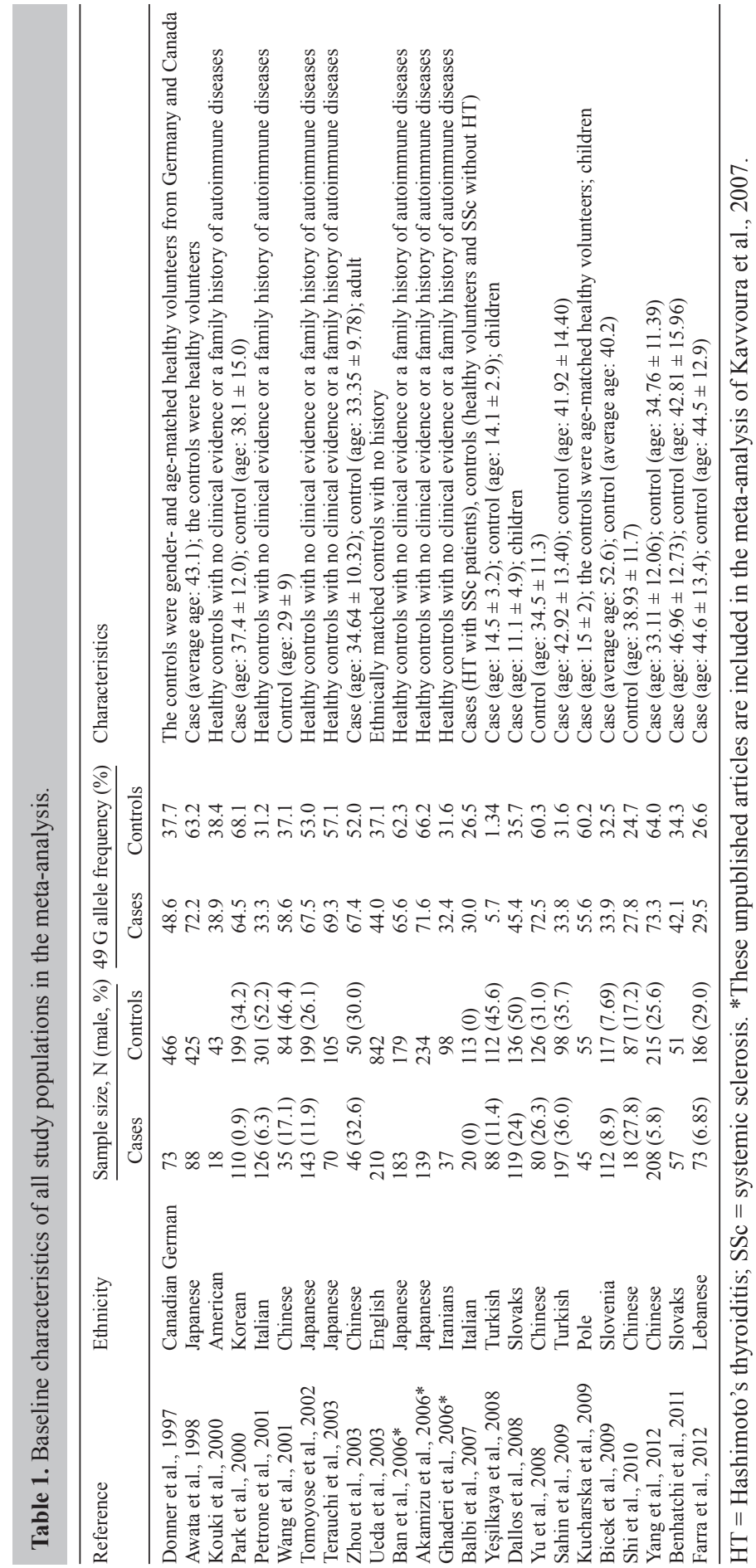




\section{Main meta-results}

We analyzed the $49 \mathrm{G}$ allele (relative to the 49A allele) and HT risk for all 24 studies (Figure 2). The inconsistency index was $44 \%$ and $\mathrm{P}$ was 0.01 , suggesting moderate heterogeneity. Thus, we chose the random-effect model to synthesize the data. Overall, comparison of alleles $49 \mathrm{G}$ and $49 \mathrm{~A}$ generated a $31 \%$ increased risk for $\mathrm{HT}(95 \% \mathrm{CI}=1.17-1.47 ; \mathrm{P}<0.00001)$. Sensitivity analyses were performed by excluding studies with cases or controls not in HWE from the overall analysis (Shi et al., 2010), and the results showed that the A49G and HT association was not significantly altered.

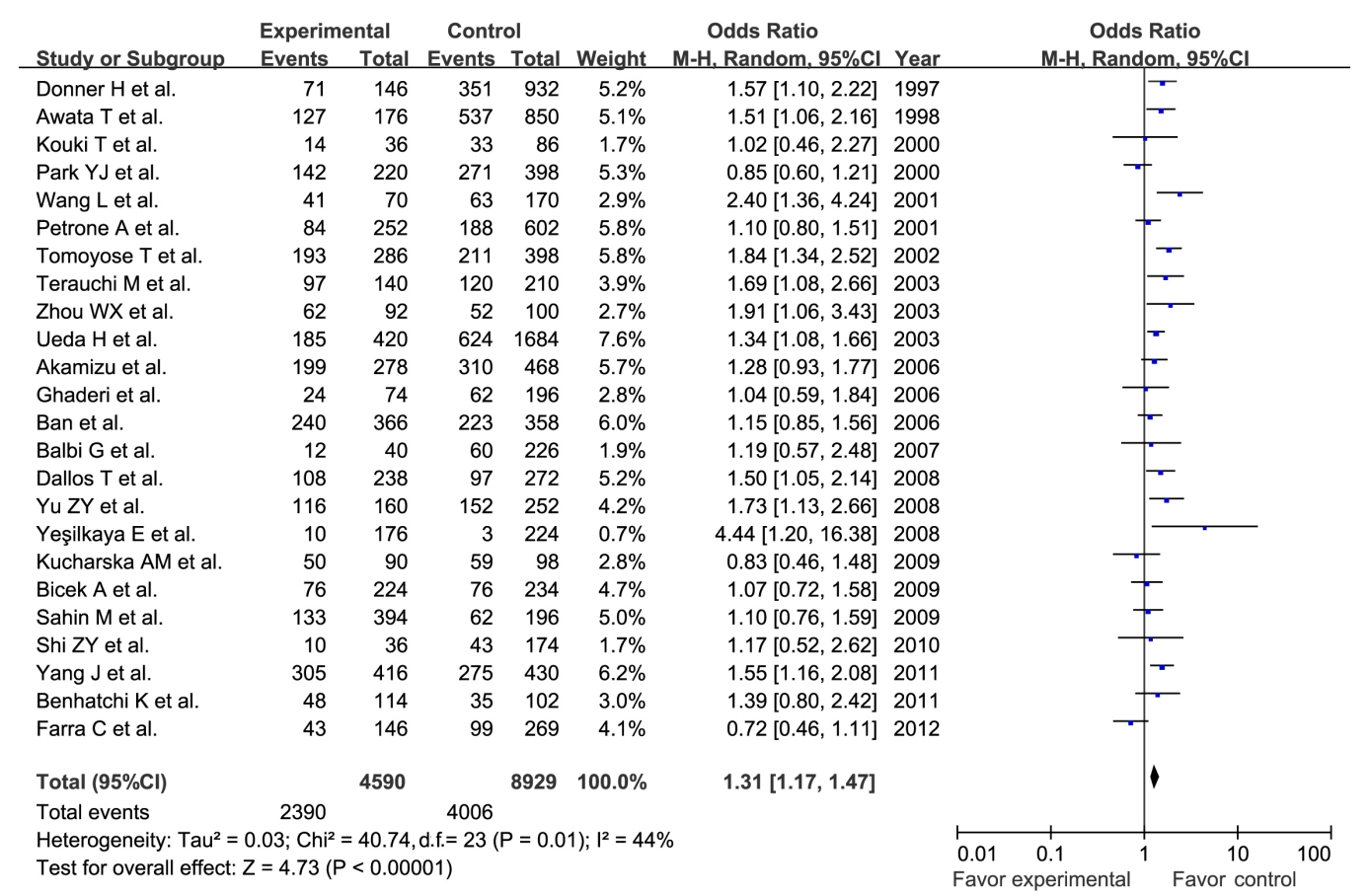

Figure 2. Pooled random-effect model based on odds ratio of Hashimoto's thyroiditis and CTLA-4 for contrasts of 49G vs 49A. M.-H. = Mantel-Haenszel estimator; 95\%CI = 95\% confidence interval; d.f. = degrees of freedom.

We performed codominant model (GG $v s$ AA and GA $v s$ AA), dominant model (GG + GA vs AA), and recessive model (GG vs GA + AA) analyses. Compared with the 49AA genotype, the 49GG and 49GA genotypes both conferred a significantly increased risk of HT. Under the dominant and recessive models, 49G allele carriers still had increased HT risk. Summary results of other genetic comparisons are listed in Table 2.

\section{Subgroup analyses}

In the subgroup analysis by ethnicity, significantly increased risks were found among East Asians $(\mathrm{OR}=1.48 ; 95 \% \mathrm{CI}=1.24-1.76 ; \mathrm{P}<0.00001)$ and whites $(\mathrm{OR}=1.27 ; 95 \% \mathrm{CI}=$ 
1.12-1.44; $\mathrm{P}=0.0002)$ but not among Middle Easterners, including Turks, Iranians, and Lebanese $(\mathrm{OR}=1.01 ; 95 \% \mathrm{CI}=0.79-1.29 ; \mathrm{P}=0.95)$, under an allele comparison (see Table 2$)$. In the subgroup analysis by age, significantly increased risks were found among adults $(\mathrm{OR}=$ $1.31 ; 95 \% \mathrm{CI}=1.17-1.47 ; \mathrm{P}<0.00001)$ but not among children $(\mathrm{OR}=1.44 ; 95 \% \mathrm{CI}=0.75$ $2.79 ; \mathrm{P}=0.27)$. Summary results of other genetic comparisons are listed in Table 2.

\section{Table 2. Summary of different comparative results.}

\begin{tabular}{|c|c|c|c|c|c|c|c|c|}
\hline Genetic model & Overall or subgroup & Study number $(\mathrm{N})$ & Participant (N) & OR $(95 \% \mathrm{CI})$ & $\mathrm{Z}$ & $P$ & $I^{2}(\%)$ & $P_{\text {het }}$ \\
\hline \multirow[t]{7}{*}{$\mathrm{G} v s \mathrm{~A}$} & All & 24 & 13519 & $1.31(1.17,1.47)$ & 4.73 & $<0.00001$ & 44 & 0.01 \\
\hline & All in HWE & 23 & 13309 & $1.35(1.21,1.51)$ & 5.25 & $<0.00001$ & 39 & 0.04 \\
\hline & Caucasians & 9 & 5796 & $1.27(1.12,1.44)$ & 3.75 & 0.0002 & 0 & 0.59 \\
\hline & The East Asians & 10 & 5838 & $1.48(1.24,1.76)$ & 4.40 & $<0.0001$ & 53 & 0.02 \\
\hline & Middle Easterns & 4 & 1675 & $1.01(0.79,1.29)$ & 0.07 & 0.95 & 60 & 0.06 \\
\hline & Children & 3 & 1098 & $1.44(0.75,2.79)$ & 1.10 & 0.27 & 68 & 0.04 \\
\hline & Adults & 20 & 12421 & $1.31(1.17,1.47)$ & 4.61 & $<0.00001$ & 42 & 0.02 \\
\hline \multirow[t]{7}{*}{ GG $v s \mathrm{AA}$} & All & 24 & 3817 & $1.94(1.60,2.36)$ & 6.65 & $<0.00001$ & 11 & 0.32 \\
\hline & All in HWE & 23 & 3733 & $1.96(1.60,2.40)$ & 6.48 & $<0.00001$ & 14 & 0.27 \\
\hline & Caucasians & 9 & 1545 & $1.72(1.31,2.26)$ & 3.91 & 0.0001 & 0 & 0.82 \\
\hline & The East Asians & 10 & 1607 & $2.48(1.71,3.60)$ & 4.79 & $<0.00001$ & 43 & 0.07 \\
\hline & Middle Easterns & 4 & 581 & $1.39(0.77,2.52)$ & 1.10 & 0.27 & 0 & 0.81 \\
\hline & Children & 3 & 398 & $1.30(0.52,3.26)$ & 0.56 & 0.57 & 57 & 0.13 \\
\hline & Adults & 20 & 3419 & $2.01(1.64,2.45)$ & 6.87 & $<0.00001$ & 5 & 0.39 \\
\hline \multirow[t]{7}{*}{ GA vs AA } & All & 24 & 5110 & $1.41(1.14,1.74)$ & 3.23 & 0.001 & 46 & 0.007 \\
\hline & All in HWE & 23 & 5021 & $1.43(1.16,1.76)$ & 3.37 & 0.0008 & 47 & 0.008 \\
\hline & Caucasians & 9 & 2397 & $1.21(0.88,1.65)$ & 1.18 & 0.24 & 51 & 0.05 \\
\hline & The East Asians & 10 & 1707 & $1.97(1.47,2.64)$ & 4.52 & $<0.00001$ & 14 & 0.32 \\
\hline & Middle Easterns & 4 & 588 & $1.19(0.49,2.87)$ & 0.38 & 0.71 & 70 & 0.04 \\
\hline & Children & 3 & 461 & $1.77(0.84,3.72)$ & 1.49 & 0.14 & 47 & 0.15 \\
\hline & Adults & 20 & 4231 & $1.43(1.12,1.83)$ & 2.82 & 0.005 & 53 & 0.004 \\
\hline \multirow[t]{7}{*}{$\mathrm{GG}+\mathrm{GA} v s \mathrm{AA}$} & All & 24 & 6444 & $1.56(1.25,1.93)$ & 4.00 & $<0.00001$ & 51 & 0.003 \\
\hline & All in HWE & 23 & 6339 & $1.58(1.27,1.97)$ & 4.07 & $<0.00001$ & 52 & 0.003 \\
\hline & Caucasians & 9 & 2790 & $1.28(0.98,1.69)$ & 1.80 & 0.07 & 44 & 0.09 \\
\hline & The East Asians & 10 & 2919 & $2.19(1.61,2.99)$ & 4.95 & $<0.00001$ & 29 & 0.18 \\
\hline & Middle Easterns & 4 & 630 & $1.27(0.59,2.71)$ & 0.61 & 0.54 & 64 & 0.06 \\
\hline & Children & 3 & 549 & $1.71(0.78,3.76)$ & 1.33 & 0.18 & 59 & 0.09 \\
\hline & Adults & 20 & 5790 & $1.57(1.24,1.99)$ & 3.72 & 0.0002 & 54 & 0.004 \\
\hline \multirow[t]{7}{*}{$\mathrm{GG} v s \mathrm{GA}+\mathrm{AA}$} & All & 24 & 6444 & $1.42(1.24,1.62)$ & 5.12 & $<0.00001$ & 3 & 0.42 \\
\hline & All in HWE & 23 & 6339 & $1.42(1.23,1.63)$ & 4.93 & $<0.00001$ & 7 & 0.36 \\
\hline & Caucasians & 9 & 2696 & $1.48(1.14,1.91)$ & 2.93 & 0.003 & 0 & 0.83 \\
\hline & The East Asians & 10 & 2919 & $1.42(1.15,1.76)$ & 3.27 & 0.001 & 41 & 0.08 \\
\hline & Middle Easterns & 4 & 630 & $1.77(0.88,3.56)$ & 1.59 & 0.11 & 0 & 0.64 \\
\hline & Children & 3 & 549 & $1.16(0.67,2.00)$ & 0.53 & 0.60 & 15 & 0.28 \\
\hline & Adults & 20 & 5790 & $1.44(1.24,1.66)$ & 4.91 & $<0.00001$ & 10 & 0.34 \\
\hline
\end{tabular}

HWE $=$ Hardy-Weinberg equilibrium; $\mathrm{OR}=$ odds ratio; $95 \% \mathrm{CI}=95 \%$ confidence interval.

\section{Publication bias}

To assess publication bias, we calculated $N_{\mathrm{fs}}$ at a significance level of 0.05 for each comparison. The $N_{\text {fs } 0.05}$ values for all contrasts were greater than the number of studies included in this meta-analysis.

\section{DISCUSSION}

Previously, Kavvoura et al. (2007) had performed a meta-analysis to investigate CTLA4 polymorphism and autoimmune thyroid diseases, and found that the $\mathrm{A} 49 \mathrm{G}$ polymorphism of 
CTLA-4 was linked to the occurrence of HT (Kavvoura et al., 2007). Several additional studies that have investigated the association between the CTLA-4 gene A49G polymorphism and HT found no link between them. The goal of this meta-analysis was to combine the results of previous studies to achieve summary conclusions about the association between the CTLA-4 gene A49G polymorphism and HT. Our meta-analysis, including 2295 patients with HT and 4521 controls, investigated the association of A49G polymorphism with HT in all studies and in ethnicity and age subgroups from the 24 studies. We included 11 novel case-control studies (Balbi et al., 2007; Dallos et al., 2008; Yesilkaya et al., 2008; Yu et al., 2008; Bicek et al., 2009; Kucharska et al., 2009; Sahin et al., 2009; Shi et al., 2010; Benhatchi et al., 2011; Yang et al., 2012; Farra et al., 2012). Furthermore, we excluded studies when the genotype distribution in controls was not in HWE (Shi et al., 2010). Although all statistical biases could not be eliminated, our results suggested that the $49 \mathrm{G}$ allele of CTLA-4 was associated with an increased HT risk.

We also carried out subgroup analysis by ethnicity and age. For ethnicity, our results showed that the $49 \mathrm{G}$ allele had a significant $27 \%$ increase for HT risk in whites and a $48 \%$ increase in East Asians after excluding studies not in HWE. Similar trends were also found under other models, with the exception of the codominant model in Caucasians. We also found no association between A49G and HT in Middle Easterners. Considering the wider confidence intervals of estimates and the small sample sizes in population-based studies, more studies are required to quantify this effect size reliably.

With respect to age, allele frequency comparison showed that the risks of HT in $49 \mathrm{G}$ allele carriers are higher in adults than in children. Other genetic model comparisons also showed a risk for HT that was higher in adults than in children.

Our study had some notable limitations. First, only published studies in English and Chinese were included, and some relevant published studies or unpublished studies with null results may have been missed, which might have biased the results. Second, owing to the relatively small number of certain eligible studies, such as the Arabs, we were unable to perform further subgroup analyses - for example by gender or other ethnicity - because of limited data. Third, some of the individual studies included a small number of cases, which may have affected the statistical power of the publication bias. Last but not the least, the potential role of the CTLA-4 A49G polymorphism may be diluted or masked by other gene-gene or gene-environment interactions. Therefore, we must refrain from drawing definitive conclusions until additional studies can confirm our results. We minimized the likelihood of bias by creating a detailed protocol before initiating our study, performing a meticulous search for publications, and using explicit methods for publication selection, data extraction, and data analysis.

Taken together, the results of this meta-analysis extended previous findings on the association between the CTLA-4 gene A49G polymorphism and HT by showing that the polymorphism was associated with an increased HT risk among whites and East Asians. Further studies should investigate adjacent markers to CTLA-4 A49G to confirm whether this association is causal or due to linkage disequilibrium. Moreover, studies of the biological mechanism and function of CTLA-4 A49G polymorphism in HT are warranted.

\section{ACKNOWLEDGMENTS}

Research supported by a grant from the National Natural Science Foundation of China (\#81071925 and \#81201837). 


\section{REFERENCES}

Almasi S, Erfani N, Mojtahedi Z, Rajaee A, et al. (2006). Association of CTLA-4 gene promoter polymorphisms with systemic sclerosis in Iranian population. Genes Immun. 7: 401-406.

Awata T, Kurihara S, Iitaka M, Takei S, et al. (1998). Association of CTLA-4 gene A-G polymorphism (IDDM12 locus) with acute-onset and insulin-depleted IDDM as well as autoimmune thyroid disease (Graves' disease and Hashimoto's thyroiditis) in the Japanese population. Diabetes 47: 128-129.

Balbi G, Ferrera F, Rizzi M, Piccioli P, et al. (2007). Association of $-318 \mathrm{C} / \mathrm{T}$ and $+49 \mathrm{~A} / \mathrm{G}$ cytotoxic T lymphocyte antigen-4 (CTLA-4) gene polymorphisms with a clinical subset of Italian patients with systemic sclerosis. Clin. Exp. Immunol. 149: 40-47.

Ban Y, Davies TF, Greenberg DA, Kissin A, et al. (2003). Analysis of the CTLA-4, CD28, and inducible costimulator (ICOS) genes in autoimmune thyroid disease. Genes Immun. 4: 586-593.

Baniasadi V, Narain N, Goswami R and Das SN (2006). Promoter region -318 C/T and -1661 A/G CTLA-4 single nucleotide polymorphisms and type 1 diabetes in North Indians. Tissue Antigens 67: 383-389.

Benhatchi K, Jochmanova I, Habalova V, Wagnerova H, et al. (2011). CTLA4 exon1 A49G polymorphism in Slovak patients with rheumatoid arthritis and Hashimoto thyroiditis-results and the review of the literature. Clin. Rheumatol. 30: 1319-1324.

Bicek A, Zaletel K, Gaberscek S, Pirnat E, et al. (2009). 49A/G and CT60 polymorphisms of the cytotoxic T-lymphocyteassociated antigen 4 gene associated with autoimmune thyroid disease. Hum. Immunol. 70: 820-824.

Dallos T, Avbelj M, Barak L, Zapletalova J, et al. (2008). CTLA-4 gene polymorphisms predispose to autoimmune endocrinopathies but not to celiac disease. Neuro Endocrinol. Lett. 29: 334-340.

Donner H, Braun J, Seidl C, Rau H, et al. (1997). Codon 17 polymorphism of the cytotoxic T lymphocyte antigen 4 gene in Hashimoto's thyroiditis and Addison's disease. J. Clin. Endocrinol. Metab. 82: 4130-4132.

Farra C, Awwad J, Fadlallah A, Sebaly G, et al. (2012). Genetics of autoimmune thyroid disease in the Lebanese population. J. Community Genet. 3: 259-264.

Kavvoura FK and Ioannidis JP (2005). CTLA-4 gene polymorphisms and susceptibility to type 1 diabetes mellitus: a HuGE Review and meta-analysis. Am. J. Epidemiol. 162: 3-16.

Kavvoura FK, Akamizu T, Awata T, Ban Y, et al. (2007). Cytotoxic T-lymphocyte associated antigen 4 gene polymorphisms and autoimmune thyroid disease: a meta-analysis. J. Clin. Endocrinol. Metab. 92: 3162-3170.

Kouki T, Sawai Y, Gardine CA, Fisfalen ME, et al. (2000). CTLA-4 gene polymorphism at position 49 in exon 1 reduces the inhibitory function of CTLA-4 and contributes to the pathogenesis of Graves' disease. J. Immunol. 165: 66066611.

Kucharska AM, Gorska E, Wasik M, Pyrzak B, et al. (2009). Expression of CD152 (CTLA-4) in children with autoimmune thyroiditis and +49 A/G polymorphism of exon 1 of the CTLA-4 gene. J. Physiol. Pharmacol. 60 (Suppl 5): 77-80.

Lee YH, Harley JB and Nath SK (2005). CTLA-4 polymorphisms and systemic lupus erythematosus (SLE): a metaanalysis. Hum. Genet. 116: 361-367.

Park YJ, Chung HK, Park DJ, Kim WB, et al. (2000). Polymorphism in the promoter and exon 1 of the cytotoxic T lymphocyte antigen-4 gene associated with autoimmune thyroid disease in Koreans. Thyroid 10: 453-459.

Petrone A, Giorgi G, Mesturino CA, Capizzi M, et al. (2001). Association of DRB1*04-DQB1*0301 haplotype and lack of association of two polymorphic sites at CTLA-4 gene with Hashimoto's thyroiditis in an Italian population. Thyroid 11: 171-175.

Sahin M, Gursoy A and Erdogan MF (2009). Cytotoxic T lymphocyte-associated molecule-4 polymorphism in Turkish patients with Hashimoto thyroiditis. Int. J. Immunogenet. 36: 103-106.

Scheipers P and Reiser H (1998). Fas-independent death of activated CD4(+) T lymphocytes induced by CTLA-4 crosslinking. Proc. Natl. Acad. Sci. U. S. A. 95: 10083-10088.

Shi ZY, Yang BZ, Wang LB, Wu RF, et al. (2010). Study of the A/G (49) polymorphism of CTLA-4 gene exon 1 in autoimmune thyroid diseases. J. Ningxia Med. Univ. 32: 330-333.

Terauchi M, Yanagawa T, Ishikawa N, Ito K, et al. (2003). Interactions of HLA-DRB4 and CTLA-4 genes influence thyroid function in Hashimoto's thyroiditis in Japanese population. J. Endocrinol. Invest. 26: 1208-1212.

Tomer Y (2001). Unraveling the genetic susceptibility to autoimmune thyroid diseases: CTLA-4 takes the stage. Thyroid 11: $167-169$.

Tomoyose T, Komiya I, Takara M, Yabiku K, et al. (2002). Cytotoxic T-lymphocyte antigen-4 gene polymorphisms and human T-cell lymphotrophic virus-1 infection: their associations with Hashimoto's thyroiditis in Japanese patients. Thyroid 12: 673-677.

Ueda H, Howson JM, Esposito L, Heward J, et al. (2003). Association of the T-cell regulatory gene CTLA4 with susceptibility to autoimmune disease. Nature 423: 506-511. 
Wang LB, Wang F, Ma RX and Yu HW (2001). Study of the A/G (49) polymorphism of CTLA-4 gene exon 1 in autoimmune thyroid diseases. Chin. Immunol. J. 17: 116-118.

Weetman AP (2003). Autoimmune thyroid disease: propagation and progression. Eur. J. Endocrinol. 148: 1-9.

Yang J, Qin Q, Yan N, Zhu YF, et al. (2012). CD40 C/T(-1) and CTLA-4 A/G(49) SNPs are associated with autoimmune thyroid diseases in the Chinese population. Endocrine 41: 111-115.

Yesilkaya E, Koc A, Bideci A, Camurdan O, et al. (2008). CTLA4 gene polymorphisms in children and adolescents with autoimmune thyroid diseases. Genet. Test 12: 461-464.

Yu ZY, Zhang JA, Maier HB, Wang Y, et al. (2008). Association of polymorphism of protein tyrosine phosphatase nonreceptor-22 gene with AITD. Xi Bao Yu Fen Zi Mian Yi Xue Za Zhi 24: 804-807.

Zhou WX, Shi BY, Wang HF and Hou MQ (2003). Association of CTLA-4 gene polymorphism with AITDs. J. Xi'an Jiaotong Univ. (Med. Sci.) 24: 170-173. 